\title{
Jonas, un passeur entre les deux rivages de la gnose et de la vie
}

\section{Natalie Depraz}

\section{OpenEdition}

\section{Journals}

Édition électronique

URL : http://journals.openedition.org/alter/291

DOI : 10.4000/alter.291

ISSN : 2558-7927

Éditeur :

Association ALTER, Archives Husserl (CNRS-UMR 8547)

\section{Édition imprimée}

Date de publication : 15 novembre 2014

Pagination : 47-62

ISBN : 978-2-9550449-0-2

ISSN : $1249-8947$

\section{Référence électronique}

Natalie Depraz, "Jonas, un passeur entre les deux rivages de la gnose et de la vie », Alter [En ligne], 22 | 2014, mis en ligne le 01 décembre 2017, consulté le 01 mai 2019. URL : http:// journals.openedition.org/alter/291; DOI : 10.4000/alter.291

Ce document a été généré automatiquement le 1 mai 2019.

Revue Alter 


\title{
Jonas, un passeur entre les deux rivages de la gnose et de la vie
}

\author{
Natalie Depraz
}

\author{
« faisant retour à mon lieu propre, la scène \\ philosophique contemporaine, après un long \\ séjour dans ces contrées lointaines, je découvris \\ que ce que j'avais appris là-bas me faisait à présent \\ mieux comprendre le rivage d'où j'étais parti. » \\ H. Jonas, « Gnose, existentialisme, nihilisme »,
} p. 217.

\section{Introduction}

1 Jonas est-il un nouveau Janus ? Un être à deux visages, qui expriment des traits contrastés et non-conciliables de sa phénoménologie, à la mesure de la gnose, communément caractérisée par la dualité du bien et du mal ? Le jeune disciple de Heidegger qui écrit Le concept de la gnose ${ }^{1}$ dès 1928 a-t-il quoi que ce soit de commun avec le biologiste du Phénomène de la vie ${ }^{2}$ hanté par la présence du vécu subjectif à la racine de la micro-vie de la cellule? Si Jonas dès sa Dissertation pose un diagnostic critique au sens d'analytique sur la gnose dans son sens le plus commun de "gnosticisme ", arrachement au monde et déficit d'incarnation, mythologie de l'âme en quête de son origine perdue, la recherche d'une subjectivité chevillée au tréfonds de la mobilité la plus incarnée du vivant suffira-telle pour autant à démontrer l'unité phénoménologique de la pensée de Hans Jonas?

2 L'objectif que je me propose dans cette contribution consiste à tenter de démentir « en première personne ", en la personne de Hans Jonas lui-même, l'imperméabilité de fait qui s'incarne aujourd'hui dans deux communautés encore très indifférentes quand ce n'est pas hostiles l'une à l'autre, celle des théologiens et celle des scientifiques. Nous avons là avec Jonas le cas assez exemplaire d'un être humain, d'une personne qui a vécu dans l'unité de sa personne deux postulations qui peuvent nous apparaître à nous, de l'extérieur, « en troisième personne », contradictoires. 
Or il y a à notre époque un enjeu intellectuel, mais aussi social et politique, à faire entrer en dialogue constructif scientifiques et porteurs de la spiritualité, et il semble que la phénoménologie soit la discipline philosophique la mieux à même de jouer ce rôle de passeur, en vertu de son aptitude à l'expérimentation et à la description, à la croisée du vécu subjectif le plus ténu et de la rigueur descriptive la plus méticuleuse. Je voudrais aujourd'hui présenter la figure de Hans Jonas comme un témoin emblématique de ce lieu charnière, propre à générer des interfaces entre des communautés intellectuelles qui ont plus que jamais besoin de se «frotter » l'une à l'autre pour nous permettre de prendre la mesure de la complexité de l'humain.

\section{Du gnosticisme à la gnose : quelle attitude phénoménologique?}

4 Les premiers travaux de Hans Jonas, c'est bien connu, portent sur le concept de gnose. Le titre de sa Dissertation, Der Begriff der Gnosis, présentée dès 1928 et avec quoi il obtient le titre de Docteur en témoigne. Tout jeune, il évolue dans le milieu sioniste, participe activement à son engagement politique, est un lecteur assidu de l'œuvre de Martin Buber, qui l'y prépare et, dès l'âge de 18 ans, il suivra à Freiburg les séminaires du fondateur de la phénoménologie, Edmund Husserl, et celui du jeune Privatdozent d'alors, Martin Heidegger, avant d'achever sa double formation théologique et philosophique à Marburg sous le double patronage de Rudolf Bultmann en théologie et de Heidegger en philosophie.

5 En septembre 1933, il fuit l'Allemagne et se réfugie à Londres, non sans avoir poursuivi son investigation sur la gnose, sous la forme d'une enquête de grande ampleur intitulée Gnosis und spätantiker Geist («La gnose et l'esprit de l'Antiquité tardive »). Deux volumes sont alors prêts, dont l'un paraîtra en 1934, intitulé Die mythologische Gnosis, dans une collection dirigée par le théologien Rudolf Bultmann ${ }^{3}$. Quant au deuxième volume, intitulé Von der Mythologie zur mystischen Philosophie, il ne pourra paraître du fait de l'instauration du régime nazi, et ne sera finalement publié qu'en $1954^{4}$.

6 Quel est l'objet du premier travail, très précoce notons-le, de Hans Jonas? Quelles sont alors ses motivations? Jonas n'a que 25 ans. Sa Dissertation, un texte de près de 500 pages, est une enquête historique de très grande ampleur, impressionnante tout autant du fait de la documentation rassemblée que de par le regard philosophique qui guide transversalement l'analyse.

7 Le noyau de son analyse critique tient en un mot, comme tel assez attendu voire galvaudé de prime abord: «dualisme». À l'encontre de la philosophie grecque platonicoaristotélicienne comme de la pensée chrétienne naissante, qui insistent chacune à leur manière sur l'unité de l'homme, du monde et de Dieu, les penseurs gnostiques développent unanimement une vision dualiste de la réalité, où l'homme et Dieu, mais aussi l'homme et le monde et enfin Dieu et le monde s'opposent par pairs comme des pôles inconciliables. C'est dans la troisième grande partie de La religion gnostique, intitulée «Le gnosticisme et l'esprit classique $»^{5}$, que Jonas instruit une comparaison structurée et minutieuse entre la philosophie grecque de l'unité harmonieuse du cosmos, la pensée chrétienne de l'unité divino-humaine de l'Incarnation et de la trine unité de la Trinité d'une part et « l'état d'esprit dualiste [qui] est le fond de toute l'attitude gnostique [...], la dualité de l'homme et du monde [allant] de pair avec celle du monde et de Dieu ${ }^{6}$. À 
premier examen, le projecteur avec lequel Jonas éclaire la pensée gnostique sous ses multiples formes n'apparaît donc guère novateur et peut même sembler assez caricatural aux yeux des spécialistes contemporains de la gnose, qui se sont attachés à faire ressortir la complexité du gnosticisme, jusqu'à retourner son dualisme apparent en une «nondualité », irréductible tout à la fois au monisme bon ton du consensus philosophicochrétien mais aussi au dualisme caricatural imputé au gnosticisme et toujours référé en dernière instance à sa figure la plus radicale, à savoir Marcion, qui concentre en lui une haine féroce de la chair et du monde ${ }^{7}$.

8 À plus ample examen, le lecteur est cependant frappé par la minutie de la description jonassienne, dans la première grande partie notamment, intitulée "La littérature gnostique: les principales doctrines. Le langage symbolique $»^{8}$, des innombrables inscriptions géographiques et culturelles de la pensée gnostique : la région de l'Euphrate où séjournèrent les Mandéens, l'Égypte avec les manuscrits coptes, le Chant de perle et l'Évangile de Vérité, l'Iran et la secte manichéenne, les manuscrits chinois du Tourfan. Plus avant, le lecteur qui pénètre à l'intérieur de la deuxième partie, intitulée «Systèmes de pensée gnostique $»^{9}$, observe avec étonnement le travail impressionnant de systématisation opéré par l'auteur pour dégager des structures cohérentes de sens pardelà l'émiettement indéfini des doctrines multiples aux narrations baroques. Par ailleurs, on ne peut que se réjouir de la neutralité scientifique tout à son honneur avec laquelle Jonas procède : il n'y a là nulle polémique, jugement de valeur, mise en cause critique ironique, ce qui, rappelons-le, caractérisa en son temps les travaux des pères grecs comme latins contemporains des gnostiques, qu'il s'agisse d'Irénée dans Contre les hérésies. La pseudo-gnose démasquée et réfutée ${ }^{10}$ ou de Tertullien dans son De carne Christi ${ }^{11}$, qui en tant qu'hérésiologues manient la rhétorique polémique de la calomnie et l'ironie à titre d'arguments critiques, en traitant par exemple les gnostiques de «chiens » et de «bêtes sauvages ". Rien de tel ici. Jonas mène une enquête historique dont l'érudition et l'exégèse analytique ne prêtent jamais le flanc au moindre soupçon de critique facile :

[...] l'enquête fut menée dans la chaleur du conflit et comme s'il y avait reconvention des plaignants. [...] Pour eux, prononcer que le gnosticisme, ce qui en lui dénaturait la vérité chrétienne, prononçait de la philosophie hellénique, c'était dresser l'acte de son accusation. Pour nous, c'est là une hypothèse parmi d'autres possibles, qui vaut comme telle, qui est pertinente au diagnostic historique du phénomène, et qu'il convient de juger selon ses mérites ${ }^{12}$.

Pour ajouter à l'esprit de neutralité scientifique dans lequel travaille l'auteur, notons qu'il n'a cessé, au gré des trois premières rééditions de l'ouvrage de 1928, à savoir en 1957, en 1962 et en 1970, de mettre à jour sa documentation, jusqu'à intégrer dans la $3^{\mathrm{e}}$ édition, la découverte des manuscrits de Nag Hammadi en 1946, sous la forme d'un chapitre intitulé "Les découvertes récentes dans le domaine du gnosticisme $»^{13}$.

Plus encore, le lecteur aiguisé remarquera, à certaines formulations de Jonas, qu'il semble par moments entrer dans un processus de valorisation de la pensée gnostique, à tout le moins pour mettre en évidence la " pertinence » de son travail de recherche, ce qui n'est bien entendu pas un défaut, loin s'en faut. Ainsi, on lit au cours de la troisième partie: "quand on le considère dans l'étendue de l'histoire universelle, la nouveauté du gnosticisme s'enlève sur son véritable fond, ce vaste monde où il est émergé, ces attitudes mentales et morales $[. ..]{\aleph^{14}}^{4}$ puis, dans l'alinéa intitulé notons-le «La réévaluation gnostique » : « le dualisme gnostique arrive comme un nouveau principe d'interprétation, s'approprie les éléments qu'il peut utiliser à ses fins et les soumet à une réinterprétation radicale $»^{15}$. Ces deux extraits, simplement indicatifs, manifestent clairement l'intérêt que 
porte l'auteur à cette pensée dite «nouvelle » et « radicale ». Faut-il pour autant conclure de l'intérêt à la fascination? Jonas a-t-il succombé à l'attraction qu'exerce immanquablement sur le chercheur l'objet de son étude lorsqu'il y consacre plusieurs années de sa vie, et ce, dès l'instant que son objectif n'est pas délibérément négatif mais garde la distance initialement neutre de l'examen critique ? Si " philosopher », comme dit si bien Wittgenstein, «c'est résister à la fascination qu'exerce sur nous certaines formes d'expression ", peut-on dire que Jonas resta ici philosophe de bout en bout ? Ou bien doiton conclure à une forme d'ambivalence même larvée faite d'attraction et de répulsion, cette ambivalence analogue qu'exerça la pensée de Martin Heidegger sur le jeune étudiant de Freiburg?

11 En tout cas, on perçoit le tropisme qu'exerce la pensée gnostique sur Jonas lorsqu'il écrit par exemple, au moment de dresser une comparaison avec le monisme qui solidarise philosophie hellénistique et christianisme (selon une visée interprétative ici fort différente des hérésiologues, qui solidarisaient quant à eux hellénisme et gnosticisme, au nom de la défense de la vérité chrétienne). Je citerai ici un peu longuement ce passage remarquablement emblématique :

Ce qui inspire la pensée gnostique, c'est la découverte angoissée de la solitude humaine au sein du cosmos, de la parfaite altérité de son être et de celui du monde entier. Cet état d'esprit dualiste est le fond de toute l'attitude gnostique ; il donne leur unité aux expressions fort diverses, plus ou moins systématiques, que cette attitude s'est données dans le rite et dans la croyance. C'est sur ce socle dualiste, sur cette expérience fondamentale passionnément vécue par l'homme, que reposent les doctrines dualistes par ailleurs distinctes.

Métaphysiquement, la dualité de l'homme et du monde va de pair avec celle du monde et de Dieu. Les termes n'en sont pas complémentaires, mais opposés. C'est une polarité d'inconciliables, et cette réalité commande l'eschatologie gnostique. La doctrine gnostique explique la dualité, ou plutôt le sentiment sur lequel elle repose, sous ses différents aspects objectifs. En théologie, on professe que le divin n'a point de part aux affaires de l'univers physique: que le vrai Dieu est rigoureusement outre mondain, que rien dans le monde ne le révèle ni même n'en donne un indice, qu'il est donc l'Inconnu, l'entièrement Autre, et qu'il n'est connaissable au moyen d'aucune analogie terrestre. En cosmologie, il est affirmé pareillement que le monde n'est pas la création de Dieu, mais de quelque principe inférieur, dont l'infériorité est une perversion du divin, et dont les traits dominants sont la domination et le pouvoir. En anthropologie, on tient que le moi intérieur de l'homme ne fait point partie du monde, création et domaine du démiurge, mais qu'au sein de ce monde, il est aussi totalement transcendant et aussi incommensurable à tous modes d'êtres cosmiques que son analogue outre mondain, le Dieu inconnu, le Dieu du dehors ${ }^{16}$.

12 Voilà une description analytique extrêmement précise, qui a pour vertu de décliner les différents plans, métaphysique, théologique, cosmologique et anthropologique où opère le gnosticisme, selon des traits descriptifs tout à fait nets: dualité polaire irréductible, inconnaissabilité et radicale altérité de Dieu, acosmisme, moi humain étranger au monde comme Dieu. Notons également que le phénoménologue fait son entrée en Jonas dès ce premier texte, quoique la démarche ne soit pas alors encore thématiquement nommée, lorsque l'auteur parle de la pensée gnostique comme d'une « attitude », et relève le mode d'être affectif fondamental de l'homme dans le monde sous les traits des tonalités de la solitude, de l'angoisse, de l'altérité radicale, et jusqu'à celle de «l'expérience fondamentale passionnément vécue par l'homme». 
13 Plus avant, Jonas nous propose à mesure une analyse affinée de ce dualisme structurel qui caractérise l'attitude gnostique, jusqu'à le faire sortir de son risque de caricature initial et en différencier plus subtilement les formes. On trouve cet affinement critique dans la Récapitulation de la fin de la deuxième partie, qui présente « les deux types de dualisme dans la spéculation gnostique »:

Des systèmes se développèrent où l'on peut distinguer deux types, que nous appelons ici pour faire court (sans nous lancer dans l'illégitime entreprise de faire la théorie d'une genèse réelle) le modèle iranien et le modèle syrien. Dans un cas comme dans l'autre, il s'agissait d'expliquer en substance les mêmes réalités, celles d'une certaine situation, conséquence d'un bouleversement métaphysique. Explication «dualiste » ici et là, et résultat commun : une fissure sépare Dieu et le monde, le monde et l'homme, l'esprit et la chair. Le modèle iranien, adaptation gnostique de la doctrine zoroastrienne, fondait ce dualisme sur deux principes opposés dès le départ; il fallait donc expliquer, avant tout, comment la Ténèbre originelle en était venue à engloutir des éléments de la Lumière : c'est-à-dire, conter le drame du monde comme une guerre aux fortunes changeantes, et montrer que la destinée divine, dont celle de l'homme fait partie et dont le monde est une conséquence indésirée, passe par le mélange et par la séparation, par la captivité et par la délivrance. La spéculation syrienne, elle, se propose la tâche plus ambitieuse de tirer la dualité elle-même et, par suite, la situation malheureuse du divin dans le système de la création, d'une seule source d'être indivise, au moyen d'une généalogie d'états divins personnifiés, émanés et déduits les uns des autres, qui marquent l'assombrissement progressif de la Lumière originelle dans les catégories de la culpabilité, de l'erreur et de l'échec. Cette "évolution descendante ", cette " dégénération » finit dans la décadence, dans cette aliénation totale de soi que représente ce monde-ci de sa passion. À la défaite et au sacrifice du divin, dans le premier cas, correspondent la culpabilité et l'erreur divines, dans le second ; à la compassion pour la Lumière devenue victime, le mépris spirituel de l'aveuglement démiurgique; à la libération finale du divin, la réformation de l'être éclairé.

Notre division est typologique, et si nous avons choisi les qualificatifs de «syrien " et d'«iranien», ce que ces mots peuvent suggérer, géographiquement et ethniquement, n'a pas grand-chose à y faire. Les systèmes valentinien et manichéen sont des exemples de l'un et de l'autre modèle. Si le principe spéculatif est différent, c'est que l'attitude religieuse, sur un fond gnostique commun, est différente, elle aussi ; et si le modèle iranien permet une mise en scène plus concrète et plus empoignante, le modèle syrien a plus de profondeur : des deux, c'est lui et lui seul qui, en conférant un statut métaphysique à la connaissance et à l'ignorance, en faisant d'elles des modes de la vie divine elle-même, peut faire pleine justice à cette réclamation du salut qui s'élève, dans toute la religion gnostique, au nom de la connaissance ${ }^{17}$.

14 Cette esquisse de typologie est extrêmement prometteuse, en ce qu'elle annonce une entente plus subtile du gnosticisme arraché à un dualisme grossier, l'ouvre à la possibilité d'une «indivision » plus profonde et à une compréhension de l'inconnaissance comme ultime connaissance plénière. C'est là que Jonas se fait le précurseur de l'entente de la gnose comme non-dualité, double négation de l'un et du deux, et situe la gnosticisme dépositaire d'une vraie et authentique gnose en forte résonnance avec les sommets de la théologie apophatique de l'Inconnaissance divine de l'Aréopagite aux IV-V $\mathrm{V}^{\mathrm{e}}$ siècles de notre ère.

15 La vertu de la description analytique jonassienne est de faire droit, dès ce texte précoce de la fin des années 20 , à une approche phénoménologique alors encore immanente, mais qui situe le propos sur un plan à la fois structurellement métaphysique et expérientiel- 
descriptif. De plus, le goût de Jonas pour l'expérience s'ajuste à la dimension affective, symbolique et narrative qui caractérise l'attitude gnostique, inscrite qu'elle est dans une histoire perméable à la dimension mythologique et plongée dans ses crises, ses drames et ses catastrophes. Ce qui donne lieu à de très belles descriptions où opère une méthode historique-structurelle, mais empreinte de la phénoménologie situationnelle et narrative concrète du drame de l'homme séparé de Dieu. Je citerai à nouveau un passage un peu long de cette même Récapitulation, au terme de la deuxième partie :

Après ce long cheminement dans le labyrinthe de la pensée et de l'imagination gnostiques, il se peut que le lecteur ait perdu de vue les grandes lignes du paysage, et qu'il trouve à propos de revenir à des vues perspectives qui lui permettront de s'orienter, même au prix de quelques répétitions.

Les gnostiques furent les premiers "théologiens" spéculatifs en ce temps où l'antiquité classique cédait la place à un nouveau phénomène religieux. La tâche leur était dictée par l'expérience gnostique fondamentale; et ceux qui étaient passés par cette expérience avaient en commun une manière d'envisager, plus ou moins a priori, la réalité. Les grands articles en étaient les notions d'un univers anti divin, de l'étrangeté de l'homme en son sein, et d'une divinité acosmique. Puisque telle était la réalité, elle présupposait une histoire explicative de cette condition « contre ». Il revenait à la spéculation d'exposer cette histoire, c'est-à-dire de rendre raison de l'état présent des choses en racontant les étapes successives de sa genèse, en partant des premiers commencements ; ce faisant, d'élever la vision de la réalité jusque dans la lumière de la gnose et d'apporter une consistante assurance de salut. Cela se fit invariablement dans la manière mythologique; mais les mythes ainsi créés, avec leurs personnifications, leurs hypostases, leur narré quasiment chronologique, sont des symboles, construits consciemment, d'une théorie métaphysique ${ }^{18}$.

On voit clairement comment Jonas a fait son miel de l'innovation de la méthode husserlienne de la description expérientielle, mais comment il a également puisé à la source heideggérienne la restitution de l'attitude affective fondamentale du gnostique. Par ailleurs, on entend ici aussi résonner la pensée cosmologique et anthropologique de Eugen Fink, strictement contemporaine de Jonas (Fink est né en 1905, il écrit la $\mathrm{VI}^{e}$ Méditation cartésienne en 1928 sous l'impulsion de Husserl, et se trouve comme Jonas déchiré entre nos deux géants à Freiburg au même moment...). On peut alors comprendre la fascination qu'exerça en son temps le maitre de Freiburg sur ces jeunes esprits brillants. On en trouve une marque (une seule, à ma connaissance) dès ce texte précoce, mais il s'agit d'un apax révélateur, qui sera d'ailleurs rappelé dans les articles ultérieurs des années 50-60 dont je vais parler dans le deuxième temps de mon propos, et qui signe son ancrage profond dans la pensée de Jonas. De quoi s'agit-il ?

Il s'agit d'une conjonction comparative notée par notre auteur à propos de la description par les gnostiques de la situation d'abandon de l'homme dans un monde sans Dieu. Jonas cite cette définition valentinienne de la gnose issue des Extraits de Théodote $(78,2)$ : « la gnose : qui étions-nous? Que sommes-nous devenus ? Où étions-nous ? Où avons-nous été jetés? Vers où nous hâtons-nous? D'où sommes-nous rachetés? Qu'est-ce que la génération? Qu'est-ce que la régénération? $»^{19}$. Et de commenter :

[...] dans la formule valentinienne [ici] citée, quoiqu'elle appartienne à une branche du gnosticisme qui explique plus volontiers la préhistoire de l'âme par ses motifs intérieurs que par une force extérieure, nous avons trouvé ces mots : «où avonsnous été jetés?» Ce mot tout concret sonne comme une note discordante après la série de verbes abstraits et neutres qui le précèdent dans la formule (être, devenir) et c'est assurément une discordance voulue. La force de choc de cette image possède elle-même une valeur symbolique dans le récit gnostique de l'existence 
humaine. Il y aurait grand intérêt à comparer l'emploi qu'en fait le gnosticisme et l'emploi qui en est fait dans une très récente analyse philosophique de l'existence, celle de Martin Heidegger. Tout ce que nous voulons dire ici, c'est que dans l'un et l'autre cas, « avoir été jeté » n'est pas simplement une description du passé, mais un attribut qui qualifie la situation existentielle telle que ce passé l'a déterminée. C'est à partir de l'expérience gnostique de la situation présentement faite à la vie que cette image dramatique de sa genèse a été projetée dans le passé, et elle fait partie de l'expression mythique de cette expérience ${ }^{20}$.

Et il ajoute en note de la troisième édition de La religion gnostique, en 1970: «sur la Geworfenheit [déjection], voir Sein und Zeit, Halle, 1927, pp. 175 et sq. J'ai tracé un parallèle des façons de voir gnostique et existentialiste dans mon article « Gnosticism and modern nihi-

lism », Social Research XIX, 4 (1952), pp. 430 et sq. ${ }^{21}$.

19 C'est ce "parallèle des façons de voir gnostique et existentialiste ", noté par Jonas luimême en 1970 depuis un point de comparaison précoce, dès 1928, autour du sens nodal de "l'être-jeté » qui cristallise et arc-boute la conjonction entre l'existentialisme heideggérien et le gnosticisme, que développera systématiquement l'article publié tout d'abord en anglais en 1952 sous le titre "Gnosticism and modern nihilism " et inclus à présent dans Le phénomène de la vie. Vers une biologie philosophique, sous le titre : «Gnose, existentialisme et nihilisme $»^{22}$. Je voudrais à présent explorer ce deuxième temps du parcours philosophique de Jonas avec la gnose, dans les années 50-60, avec l'idée d'une continuité forte avec le travail des années 20 , ce dernier ayant été à chaque nouvelle édition relu, revu et actualisé, et ce jusque dans les années 70 .

\section{De la vie à l'existence, et retour à la vie : passages phénoménologiques génératifs entre gnose et existentialisme}

Dans cet article (et quelques autres de la même période que, par économie, je ne ferai ici que mentionner $\left.{ }^{23}\right)$, réinscrit ici dans un ensemble d'articles écrits et de conférences prononcées entre 1951 et 1973 avec pour centre de gravité "le phénomène de la vie ", l'ancien étudiant de Heidegger se livre à un diagnostic comparatif expérimental entre deux situations historico-anthropologiques. Tout en prenant fortement appui sur l'enquête historique initiale, tout en ayant précocement noté l'être-jeté comme point de cristallisation entre les deux attitudes, le propos méthodologique et ontologique a changé de dimension. Il ne s'agit plus de se livrer - ainsi le thématise à présent Jonas rétrospectivement - à une "lecture existentialiste de la gnose ", comme il s'est agi de le faire dans le texte des années 20 , sur un mode disons-le immanent c'est-à-dire nonrevendiqué, mais de procéder davantage à une "lecture gnostique de l'existentialisme ». C'est en tout cas de cette façon que, à l'orée de l'article de $1952^{24}$, Jonas décrit le double mouvement méthodologique herméneutique réciproque de sa trajectoire phénoménologique avec la gnose aux deux grands moments de sa pensée :

les fonctions herméneutiques s'inversent, et deviennent réciproques - la serrure devient clé et la clé serrure : la lecture «existentialiste » de la Gnose, si bien justifiée par son succès herméneutique, invite comme à son complément naturel à tenter une lecture « gnostique » de l'existentialisme ${ }^{25}$. 
21 deux courants de pensée co-opèrent en se co-instrumentalisant l'un l'autre et finissent par jouer l'un par rapport à l'autre le rôle double et mutuel d'une méthode d'accès et d'une attitude expérientielle. D'où l'usage fort du terme de "réciprocité ", thématisé comme méthode d'« éclairage réciproque des deux », et nommé « circularité » en un sens ici vertueux. On a affaire à mon sens à un sens novateur de l'herméneutique, qui n'est plus ni exégèse textuelle au sens standard $d u$ terme, ni non plus simplement herméneutique de la compréhension facticielle au sens heideggérien (même si elle y emprunte la posture de base), ni non plus herméneutique du dialogue au sens gaddamérien (même si la réciprocité des deux mouvements l'un devenant l' interpretandum de l'autre répond à la dynamique relationnelle imprimée par Gaddamer). Il y a dans l'herméneutique jonassienne un geste proto-éthique de dépossession de l'interprète à l'égard de son propre pouvoir d'interprétant, toujours dépositaire d'une vérité apposée sur le réel à comprendre. Ici, gnosticisme et existentialisme deviennent les vérités l'une de l'autre, de sorte que leur pouvoir de vérité est renvoyé dos-à-dos et que domine alors la seule vérité d'une expérience, celle d'un homme jeté dans le monde, abandonné, angoissé, solitaire, dont gnosticisme et existentialisme décrivent deux situations anthropologiques historiquement ciblés, tout à la fois indicatives et exemplaires, mais en tout cas ni explicatives, ni exclusives. Ce geste de double lecture croisée répond à mon sens davantage à une ontologie co-générative d'émergence du sens qu'à une herméneutique, où j'ai du mal à voir autre chose qu'une imposition de sens et un geste unilatéral de domination d'une vérité qui assigne son sens ${ }^{26}$. Certes, Jonas continue à emprunter au contexte philosophique ambiant le terme "herméneutique ", mais il me semble qu'il signifie en réalité une autre posture en parlant de "comparaison » «à titre expérimental », de "circularité », d'« éclairage réciproque ». Notamment, l'énoncé de sa méthodologie d'entrée de jeu montre qu'il travaille en structure dynamique et en régime de co-émergence de sens, et non en filiation historique et en explicitation d'impensés ${ }^{27}$. On est très loin ici de l'herméneutique au sens fort. Relisons le premier paragraphe de l'article : « dans cet essai, je me propose, à titre expérimental, d'établir une comparaison entre deux mouvements, deux positions ou deux systèmes de pensée largement séparés dans le temps et l'espace, et apparemment incommensurables à première vue $»^{28}$. Ce qui n'empêche pas l'auteur de rester prudent sur l'usage de cette méthode, en soulignant « les réserves qu'appelle le caractère expérimental d'une comparaison de ce genre " ${ }^{29}$.

Dans le format d'un témoignage en première personne où il fait retour sur son parcours intellectuel, «ma propre expérience ", Jonas fait apparaître avec transparence et souci de clarté la co-implication réciproque de ces deux intérêts :

[...] l'existentialisme, qui avait fourni les moyens d'une analyse historique, se trouvait lui-même impliqué dans les résultats de celle-ci. [...] ses catégories convenaient comme si elles étaient faites sur mesure. [...] l'applicabilité des catégories dans le cas donné [était] dû au genre même d'« existence» de part et d'autre - celui qui avait fourni les catégories et celui qui leur correspondait si bien ${ }^{30}$

Il reconnait avoir été alors "attiré " "dans le labyrinthe gnostique » en vertu d'une « affinité obscurément ressentie » entre « l'optique » « acquise à l'école de Heidegger » et les aspects de l'attitude gnostique alors si aisément discernés. d'éclairer l'existentialisme par le gnosticisme ou, de façon plus neutre, de manifester le 
troublant « cousinage » entre deux situations historiques, celle des trois premiers siècles du Christianisme, celle de l'existentialisme moderne, situation de l'homme désorienté, ininscrit dans un cosmos unifié dont il ferait pleinement partie, doté d'un sentiment de solitude, d'isolement, de ce que Jonas note comme étant un "acosmisme anthropologique $»^{31}$. Ou bien : Jonas par sa mise en perspective comparative générative ne révèlerait-t-il par contre coup un Heidegger " gnostique " ? Y aurait-t-il là critique, même indirecte, du refus heideggérien du corps?

Rien n'est moins sûr : la lecture de l'article de 1952 fait davantage ressortir la fascination continuée de Jonas à l'égard du Heidegger de Être et temps (1927), et ce, en dépit du rejet de ce dernier dès 1933 lorsqu'il apprend sa complicité avec le régime nazi. Le propos est en effet ponctué par des références enthousiastes à l'ontologie fondamentale: c'est d'abord l'interprétation de l'attitude gnostique en termes de Stimmung affective d'étrangeté au monde ${ }^{32}$, d'abandon de l'homme, de son altérité solitaire et de son sentiment d'angoisse ${ }^{33}$; c'est ensuite le nihilisme commun qui caractérisent les deux attitudes et définit via Nietzsche la mort de Dieu et du monde comme un enfoncement dans le vide et l'abyme du rien, où le Dieu caché est transcendance sans pouvoir efficient et le soi acosmique ${ }^{34}$; c'est surtout le cinquième et dernier point de l'article ${ }^{35}$, qui donne toute sa place à l'ouvrage de 1927 comme «ce manifeste le plus profond et jusqu'ici le plus important de la philosophie existentialiste »: Jonas y remarque l'importance des « catégories de mouvements » que sont les existentiaux, non plus catégories objectives de la réalité comme chez Kant, mais "structures primaires de la réalisation", «structures fonctionnelles du mouvement actif du temps interne par lequel un "monde" est maintenu et est donné naissance au soi comme événement continuel $\aleph^{36}$. À propos de cette "signification profondément temporelle" des existentiaux, catégories d'un nouveau genre qui articulent le temps interne de l'existence dans ses tensions, Jonas dit faire une "découverte frappante", qui révèle selon lui la portée révolutionnaire de l'analyse heideggérienne : sous la rubrique "présent ", à savoir "existence authentique ", nous n'avons dans la table des catégories reconstituées pour l'occasion qu'une case « vide ». Et Jonas de donner une signification forte à ce vide, peut-être plus encore que Heidegger luimême: le vide manifeste la césure de "l'instant» (Augenblick), comme tel invisible, véritable "blanc d'antenne ", à jamais inassimilable. Dans les termes de Jonas, le présent est situation entièrement définie comme relation du soi à son avenir et à son passé : « il passe comme un éclair ", dit Jonas. À ce titre, le nom du présent, «l'instant ", tout en étant dynamique incessante et mouvement interne, n'est rien de la durée. Il tient dans cette tension maintenue, et s'il est séparé de sa dynamique, il retombe dans l'inauthentique de la vie objective, le faux présent comme dit Jonas de la Verfallenheit. Je ne résiste pas au plaisir de citer ici cette formule si incisive : «il n'y a point de présent où habiter, seulement la crise entre passé et avenir, l'instant pointu de l'intervalle, en équilibre sur le fil du rasoir de la décision qui pousse en avant $»^{37}$. On a là une synthèse chatoyante remarquable du micro-présent vivant husserlien, de l'événement décisionnel heideggérien, mais aussi comme une anticipation de l'écart diachronique lévinassien, altérité du soi dans sa réappropriation possible, à quoi le vocabulaire de la crise, du pointu et du rasoir, proprement jonassien, fait plus écho que celui de ses deux maîtres de Freiburg, et qui consonne d'ailleurs fortement avec l'altérité radicale qui habite à son tour le gnostique en crise interne de son soi altéré. de sa situation historique de témoin "fasciné", on serait en droit de s'inquiéter. Mais 
voilà, l'auteur du Principe responsabilité, s'il n'est pas né encore, est déjà à l'œuvre. Écoutons-le :

Ce dynamisme à perdre haleine exerçait une séduction formidable sur l'esprit contemporain, et ma génération dans les années vingt et au début des années trente en Allemagne y succomba en masse. Mais il y a une énigme dans cette évanescence du présent comme détenteur d'un contenu véritable, dans sa réduction au point zéro inhospitalier de la simple résolution formelle. Quelle situation métaphysique se tient là-derrière ${ }^{38}$ ?

Et Jonas d'amorcer une entente différente du présent, point plénier de déhiscence et d'émergence, lieu-source plutôt que vide, jaillissement plutôt zéro hostile. On comprend ici pourquoi Heidegger n'est pas un philosophe de la surprise, et pourquoi Jonas, lui, l'est 39. À cet égard, même la reprise de la Geworfenheit heideggérienne, à la page précédente, se voit réévaluer dans un vocabulaire qui n'est pas celui de Heidegger ni non plus d'ailleurs du gnosticisme, mais celui de la dynamique et de la vie :

Mais l'image du jeter donne aussi un caractère dynamique au tout de l'existence ainsi entamée. Dans notre formule, ce caractère est repris par l'image de la course vers une fin (end). Ejectée dans le monde, la vie est un genre de trajectoire se projetant soi-même vers l'avant dans l'avenir ${ }^{40}$.

On comprend alors le statut du point 4, qui relativise l'enthousiasme de Jonas, fait jouer l'alternative sartrienne et entre en discussion critique avec la conception heideggérienne de l'animalité. En effet, le co-éclairage de la gnose et de l'existentialisme dépasse la référence à Heidegger : la thématique de l'existence authentique et de la liberté est celle du Sartre de L'existentialisme est un humanisme ${ }^{41}$, repris à son compte par Jonas, tandis qu'il se déprend du Heidegger de la Lettre sur l'humanisme et y critique la conception étroite de l'animalité ${ }^{42}$ ainsi que le rejet de la nature ${ }^{43}$. Il y a là un levier critique qui aura d'avance balayé ce que le point $\mathrm{v}$ laisse transparaître d'enthousiasme. Le jeune disciple fasciné cède la place au penseur de la vie et du corps. Le Heidegger gnostique est démasqué, et si Jonas dénonce selon le geste du maître "l'oubli de la vie », écho de "l'oubli de l'être », c'est pour mieux en renverser les attendus : dans la pensée moderne, la vie est mise entre parenthèses, la pensée écartelée entre deux extrêmes, idéalisme acosmique et matérialisme de la matière morte. La vie est mise en concepts, elle n'est pas éprouvée, et surtout pas par Heidegger, qui consonne avec le gnosticisme dans les traits critiquables de haine du corps organique et de la nature ${ }^{44}$. Ainsi, contrairement au gnosticisme et à ses avatars modernes, Évolution et liberté nous enjoint à prendre au sérieux l'organique. Il s'agit de ne surtout pas le diaboliser, de ne pas le considérer comme inférieur ou comme le mal ; l'organique, dans ses manifestations les plus élémentaires, pré-figure (volbildet) le spirituel; en effet l'organique, dans la profusion de ses formes de vie, dans son imprévisibilité, sa réserve inépuisable de "surprises », est le creuset éminent de notre libertét5.

29 C'est à partir de ce socle de la libre surprise existentielle - spirituelle - inhérente à l'organicité du vivant que Jonas apparaît comme un passeur emblématique, à même de co-générer des liens entre des communautés religieuse et scientifique encore trop hermétiques l'une à l'autre et qu'il est urgent aujourd'hui de faire se rencontrer de façon à pouvoir prendre la pleine mesure de la complexité de l'humain. 


\section{NOTES}

1. H. Jonas, Der Begriff der Gnosis, Dissertation sous la direction de Heidegger, Marbourg, 30 septembre, Göttingen, Hubert, 1930, 52 p., actuellement indisponible. Voir à ce propos « Der Begriff der Gnosis », « Methodologische Einleitungen » $(1927,1930)$ publiées in C. Bonaldi, ed., «Conoscere Dio », Milan, Albo Versorio, 2006.

2. H. Jonas, The Phenomenon of Life. Toward a Philosophical Biology, New York, Harper \& Row, 1968, Le phénomène de la vie. Vers une biologie philosophique, trad. fr. D. Lories, Bruxelles, De Boeck, 2001.

3. H. Jonas, Gnosis und spätantiker Geist, volume 1 : Die mythologische Gnosis. Mit einer Einführung zur Geschichte und Methodologie der Forschung (1934), Göttingen, Vandenhoeck \& Ruprecht, $4^{\mathrm{e}}$ édition, 1988.

4. H. Jonas, Gnosis und spätantiker Geist, volume 2 : Von der Mythologie zur mystischen Philosophie, Göttingen, Vandenhoeck \& Ruprecht, 1954 (publication partielle), 3 ème édition complétée par Kurt Rudolph, 1993 ; H. Jonas, Die gnostische Religion : die Botschaft des fremden Gottes und die Anfänge des Christentums, Insel Verlag, 1988; publications anglaise et française préalables, La religion gnostique : le message du Dieu étranger et les débuts du Christianisme, trad. angl. en 1958 ; trad. fr. en 1978 par L. Evrard, Paris, Flammarion, 1992, 506 p.

5. H. Jonas, La religion gnostique, op. cit., pp. 315-370.

6. Ibid., p. 330.

7. P. Ioan Couliano, Les gnoses dualistes d'Occident: histoire et mythes, Paris, Plon, 1990, et N. Depraz, «Le statut phénoménologique du monde dans la gnose : du dualisme à la non-dualité ", Laval théologique et philosophique, vol. 52, $\mathrm{n}^{\circ}$ 3, 1996, pp. 625-647. Cf. aussi N. Depraz et J.-F. Marquet éds, La gnose, une question philosophique ? Pour une philosophie de l'invisible, Paris, Cerf, 2000.

8. H. Jonas, La religion gnostique, op. cit., première partie, pp. 50-130.

9. H. Jonas, La religion gnostique, op. cit., deuxième partie, pp. 130-311.

10. Irénée de Lyon, Contre les hérésies (180), Paris, Cerf, 2006.

11. Tertullien, De la chair du Christ, Paris, Cerf, 1975.

12. H. Jonas, La religion gnostique, op. cit., Préface (1957), p. 9.

13. Ibid., chapitre XII, pp. 377-415.

14. Ibid., p. 315.

15. Ibid., p. 340. Je souligne.

16. Ibid., p. 330. Je souligne.

17. Op. cit., p. 312.

18. Ibid., p. 312.

19. Ibid., p. 68.

20. Ibid., pp. 91-92.

21. Cet article de 1952 a été publié à titre d'Épilogue à la troisième édition de La religion gnostique, op. cit., pp. 417-442.

22. H. Jonas, Le phénomène de la vie. Vers une biologie philosophique, op. cit., pp. 217-237.

23. H. Jonas, op. cit., Essai X, «Heidegger et la théologie» (1964), pp. 239-262 ; «Le syndrome gnostique : typologie d'une pensée, d'une imagination et d'une sensibilité », Contribution au Colloque international sur «Les origines du gnosticisme », Messine, avril 1966, publié d'abord sous le titre "Delimitation of the Gnostic Phenomenon - Typological and Historical », dans Le origini del Gnosticismo, éd. Ugo Bianchi (Leyde, EJ. Brill, 1967), reproduite dans Hans Jonas, Philosophical Essays, Englewood Cliffs, Prentice-Hall, Inc., 1974, sous le titre "The gnostic 
Syndrom : Typology of its Thought, Imagination, and Mood ", enfin publié comme appendice à $L a$ religion gnostique, op. cit., pp. $443 \mathrm{sq}$.

24. H. Jonas, Le phénomène de la vie. Vers une biologie philosophique, op. cit., pp. 217-218.

25. Ibid., p. 218.

26. Cf. ma mise à distance de l'herméneutique dès Transcendance et Incarnation. L'altérité à soi comme intersubjectivité chez E. Husserl, Paris, Vrin, 1995, Introduction.

27. C'est exactement la méthode que j'ai employée pour faire travailler ensemble selon une cogénérativité $\mathrm{du}$ sens sans filiation historique avérée les démarches phénoménologique et philocalique, sans me référer alors à Hans Jonas car je n'avais pas en tête cet article, dans $\mathrm{N}$. Depraz, Le corps glorieux. Phénoménologie pratique de la Philocalie des Pères du désert et des Pères de l'Église, Bruxelles, Bibliothèque philosophique de Louvain, 2008, Introduction.

28. Ibid., p. 217

29. Ibid., p. 222.

30. Ibid., p. 218.

31. Ibid., p. 222.

32. Ibid., p. 223.

33. Ibid., p. 225.

34. Ibid., p. 229.

35. Ibid., pp. 232-236.

36. Ibid., pp. 233-234.

37. Ibid., p. 235 pour la citation ; pp. 234-235 pour l'analyse.

38. Ibid., p. 233.

39. Pour plus de détails sur ce point, cf. N. Depraz, « La surprise du vivant. Levinas et MerleauPonty à la lumière de Hans Jonas ", in L'héritage de la phénoménologie et le problème de la vie, a c. di E. Ferrario, Rome, Lithos editrice, 2012 ; «Phenomenology of surprise», in T. Nenon \& Ph. Blosser eds., "Advancing Phenomenology, Essays in Honor of Lester Embree ", Springer, Contributions to Phenomenology 62, 2010, pp. 223-235. Plus largement, voir l'ANR Emphiline « La surprise au sein de la spontanéité des émotions : un vecteur de cognition élargie ", que je dirige aux Archives-Husserl (équipe pilote, page sur le site des Archives).

40. H. Jonas, Le phénomène de la vie, op. cit., p. 233.

41. Ibid., p. 230.

42. Ibid., p. 231.

43. Ibid., p. 232.

44. Ibid., p. 235.

45. H. Jonas, Évolution et liberté, Paris, Éditions Rivages, 2005. 